\title{
Unsteady Simulation of Turbulent Axial Vortex by Means of a
}

\author{
Non-Linear $k-\varepsilon$ Model \\ Md. Shahjahan Ali *, Takashi Hosoda **, Ichiro Kimura*** \\ * PhD Student, Department of Urban Management, Kyoto University (615-8540, Kyoto) \\ ** Professor, Department of Urban Management, Kyoto University (615-8540, Kyoto) \\ ***Associate Professor, Faculty and Graduate School of Engineering, Hokkaido University, (060-8623, Sapporo)
}

\begin{abstract}
Unsteady numerical simulation is carried out for 3D velocity field of a turbulent trailing vortex using non-linear $k-\varepsilon$ model. The general features of vortex decay as well as the different time periods of turbulence growth/decay captured in present simulation are discussed. The self similarity in the radial distribution of tangential velocity and circulation profiles are examined for trailing vortex. The simulation is also performed using standard $k-\varepsilon$ model, and comparison is shown with non-linear model. Since the rotational effects of vortex were not captured by a standard $k-\varepsilon$ model, it predicted extremely rapid and strong turbulence growth and causes a rapid decay of tangential and axial velocities. However, the non-linear model shows good agreement with previous DNS data. It is observed that the decay rate of axial velocity is much higher than tangential velocity. Five different time zones are observed in the growth/decay process of turbulent kinetic energy. In the matured stage of a trailing vortex, the turbulent normal stresses show two types of anisotropic behavior depending on the radial distance.
\end{abstract}

Key Words: Axial vortex, Trailing vortex, Rankine vortex, RANS, non-linear $k-\varepsilon$ model.

\section{Introduction}

The basic types of plane vortices can be classified into two categories: one is slower velocity at center and maximum at sides; and another is maximum at center and minimum velocity at edges. The rotary fluid motion of first one is called the solid body rotation, since it is similar to the fluid motion filled in a rotating hollow box. On the other hand, if a long circular rod rotates in a fluid with constant velocity around its axis, the fluid velocity can be found highest and equal to the velocity of rod at the rods surface (due to adhesion); and with increasing distance from the rod, the velocity is observed to be diminished in inverse proportional to the distance. Such a fluid motion is called a potential vortex. Fluid motion composed of a potential vortex and solid body rotation is called Rankine vortex after the fluid dynamicist Rankine. The radial distance from center to the maximum tangential velocity is called the radius of vortex core. For a steady circular motion without a velocity component normal to the plane of rotation, the Rankine vortex in the only possible vortex whose velocity is zero at the center as well as far away from it. In addition to these basic vortices, there are other time dependent rotary motions that have azimuthal velocity component as well as radial and axial components.

The existence of such vortices is not limited to natural activities but also in many engineering applications. The Rankine vortex has been used extensively in various studies; for instance, to predict the decay of wing-tip vortices, to estimate the noise level produced by vortices and vibrations, to model the natural phenomena such as hurricanes and tornados. Current aircraft spacing in and around airports is partially governed by the vortex wake hazard caused by the possible interaction of leader aircrafts trailing wake and vortices with a following aircraft. However, the vortex behavior behind a lifting vehicle is still a topic of debate. The understanding about the internal structure as well as the mechanism of turbulence growth in the vortex core is also debatable. Vortex flows are the major cause of cavitation and underwater acoustics in marine propellers. Cavitation and hollow core due to pressure deficit at vortex center causes air-entraining vortices, which is an important part in 
hydraulics. Therefore, the emphasis on present topic is not only due to academic interest, but due to its practical importance as well.

This paper deals with the trailing vortex, which is a time dependent vorticity concentration created at the tip of a semi-infinite wing with two non-zero velocity components, $V_{\theta}$ (tangential) and $V_{z}$ (axial), and constant circulation at infinity. Therefore, the main difference between the trailing and line vortex is the presence of axial velocity component.

It is well known that the RANS (Reynolds Averaged Navier Stokes) type turbulence models, such as two equation model or Reynolds stress model, are the most popular tool used for practical engineering applications ${ }^{1)}$. Because, it requires less CPU time and computer memory compared to LES and DNS. Therefore, the clarification of the possibility, the limitation and areas of improvement of RANS models should be still paid attention to. To resolve the Reynolds stress term appeared in the averaged Navier-Stokes equations, the $k-\varepsilon$ model is one of the most frequently adopted one ${ }^{1)}$. However, the standard $k-\varepsilon$ model cannot produce satisfactory results for the flow field having high rate of strain and rotation because of its isotropic assumption of eddy viscosity ${ }^{2}$. On the other hand, a non-linear model predicts superior result by capturing the anisotropic turbulence. The non-linear model used in this study differs from the standard $k-\varepsilon$ model in two important ways: i) the eddy viscosity coefficient $\left(c_{\mu}\right)$ is not a constant but a function of strain and rotation parameters; and ii) non-linear terms are added in the Reynolds stress equation to account the anisotropy of normal stresses. The model constants are tuned considering the realizability conditions and the anisotropy of turbulence in simple shear flows (Ali et al. ${ }^{3), 4)}$ ).

In this study, unsteady numerical simulation with 3D velocity field is carried out for a turbulent axial vortex using non-linear $k-\varepsilon$ model. The simulation is also performed using standard $k-\varepsilon$ model, and the performance of the models are assessed by comparing the simulated results with previous DNS data. The general features of vortex decay as well as the different time zones of turbulence growth/decay captured in present simulations are discussed. The self similarity in radial distribution of tangential velocity and circulation profiles are examined for trailing vortex.

In previous studies, the turbulent trailing vortex forming from a rolling up vortex sheet have been studied extensively both in experimentally and numerically. The experimental results and theoretical observation by Phillips ${ }^{5)}$ and the DNS numerical study by Qin $^{6)}$ are considered here to compare the non-linear $k-\varepsilon$ model predictions. Phillips ${ }^{5)}$ described the Rankine vortex as a multi-layered structure. The innermost region is called as vortex core (Region-I). The tangential velocity is zero at the center of the core where viscosity produces nearly solid body rotation. The outer most region (Region-III) can be described by the wake diffusion but extended inward to include turbulent diffusion. The Region-II is a buffer region between the nearly potential outer flow and the solid body rotation of core. This region contains the point of maximum tangential velocity where the inertial effects are negligible but high strain rate exist. Hoffmann \& Joubart ${ }^{7)}$ reported that this region is very similar to that of turbulent boundary layer near wall, and believed to follow a behavior of log law.

\section{Non-Linear $k-\varepsilon$ Model}

\subsection{Basic equations}

The basic equations in a $k-\varepsilon$ model for an unsteady incompressible flow are as follows.

Continuity equation:

$$
\frac{\partial U_{i}}{\partial x_{i}}=0
$$

Momentum equation:

$\frac{\partial U_{i}}{\partial t}+\frac{\partial U_{j} U_{i}}{\partial x_{j}}=g_{i}-\frac{1}{\rho} \frac{\partial P}{\partial x_{i}}+\frac{\partial}{\partial x_{j}}\left(-\overline{u_{i} u_{j}}\right)+v \frac{\partial^{2} U_{i}}{\partial x_{j}^{2}}$

$k$ - equation:

$\frac{\partial k}{\partial t}+\frac{\partial k U_{j}}{\partial x_{j}}=-\overline{u_{i} u_{j}} \frac{\partial U_{i}}{\partial x_{j}}+\frac{\partial}{\partial x_{j}}\left\{\left(\frac{v_{t}}{\sigma_{k}}+v\right) \frac{\partial k}{\partial x_{j}}\right\}-\varepsilon$

$\varepsilon$ - equation:

$\frac{\partial \varepsilon}{\partial t}+\frac{\partial \varepsilon U_{j}}{\partial x_{j}}=-c_{\varepsilon 1} \frac{\varepsilon}{k} \overline{u_{i} u_{j}} \frac{\partial U_{i}}{\partial x_{j}}+\frac{\partial}{\partial x_{j}}\left\{\left(\frac{v_{t}}{\sigma_{\varepsilon}}+v\right) \frac{\partial \varepsilon}{\partial x_{j}}\right\}-c_{\varepsilon 2} \frac{\varepsilon^{2}}{k}$

where, $x_{i}$ : the spatial coordinates, $U_{i}$ and $u_{i}$ : the average and turbulent velocities respectively in $x_{i}$ direction, $\mathrm{P}:$ the pressure, $\rho$ : the density of fluid, $v$ : the molecular kinematic viscosity, $k:$ the averaged turbulent energy, $\varepsilon$ : the averaged turbulent energy dissipation rate, $v_{t}:$ the eddy viscosity, $\sigma_{k}, \sigma_{\varepsilon}, c_{\varepsilon l}, c_{\varepsilon 2}$ : the model constants (standard values of $\sigma_{k}=1.0, \sigma_{\varepsilon}=1.3, c_{\varepsilon l}=1.44$ and $c_{\varepsilon 2}=1.92$ are used) ${ }^{2)}$.

\subsection{Constitutive equations}

(a) Standard $k-\varepsilon$ model

In the standard $k-\varepsilon$ model, the Reynolds stress tensor $\overline{u_{i} u_{j}}$ is solved by linear constitutive equations derived from Boussinesq eddy viscosity concept, which does not take into account the anisotropy effect. 
$-\overline{u_{i} u_{j}}=v_{t} S_{i j}-\frac{2}{3} k \delta_{i j}, \quad S_{i j}=\frac{\partial U_{i}}{\partial x_{j}}+\frac{\partial U_{j}}{\partial x_{i}}$

Here, $v_{t}$ is determined from the dimensional consideration of $k$ and $\varepsilon$, and is approximated by

$v_{t}=c_{\mu} \frac{k^{2}}{\varepsilon}$.

Here, $c_{\mu}$ bears a constant value of 0.09 .

(b) Non-linear $k-\varepsilon$ model

Including the non-linear anisotropy term in the Reynolds stress equation introduced by Yoshizawa ${ }^{8)}$, the constitutive equations can be expressed in the following forms

$-\overline{u_{i} u_{j}}=v_{t} S_{i j}-\frac{2}{3} k \delta_{i j}-\frac{k}{\varepsilon} v_{t} \sum_{\beta=1}^{3} c_{\beta}\left(S_{\beta i j}-\frac{1}{3} S_{\beta \alpha \alpha} \delta_{i j}\right)$

Here, $v_{t}$ is also determined as Eq. (6), but $c_{\mu}$ is not a constant; $c_{\beta}$ is the coefficient of non-linear quadratic term; and $S_{\beta i j}$ are defined as follows:

$$
S_{1 i j}=\frac{\partial U_{i}}{\partial x_{\gamma}} \frac{\partial U_{j}}{\partial x_{\gamma}}, S_{2 i j}=\frac{1}{2}\left(\frac{\partial U_{\gamma}}{\partial x_{i}} \frac{\partial U_{j}}{\partial x_{\gamma}}+\frac{\partial U_{\gamma}}{\partial x_{j}} \frac{\partial U_{i}}{\partial x_{\gamma}}\right),
$$

and $S_{3 i j}=\frac{\partial U_{\gamma}}{\partial x_{i}} \frac{\partial U_{\gamma}}{\partial x_{j}}$.

It is known that the non-linear terms in equation (7) are equivalent to the following mathematical formulation ${ }^{9), 10)}$.

$\alpha_{1}\left(S_{i l} \Omega_{l j}+\Omega_{l i} S_{j l}\right)+\alpha_{2}\left(S_{i l} S_{l j}-\frac{1}{3} S_{k m} S_{m k} \delta_{i j}\right)$

$+\alpha_{3}\left(\Omega_{i l} \Omega_{l j}-\frac{1}{3} \Omega_{k m} \Omega_{m k} \delta_{i j}\right)$

Where, the strain and rotation tensors are defined as

$S_{i j}=\frac{\partial U_{i}}{\partial x_{j}}+\frac{\partial U_{j}}{\partial x_{i}}, \Omega_{i j}=\frac{\partial U_{i}}{\partial x_{j}}-\frac{\partial U_{j}}{\partial x_{i}}$

Comparing Eq. (9) with the non-linear terms of Eq. (7), the relations between the coefficients can be derived as

$c_{1}=-2 \alpha_{1}+\alpha_{2}-\alpha_{3}, c_{2}=2\left(\alpha_{2}+\alpha_{3}\right), c_{3}=2 \alpha_{1}+\alpha_{2}-\alpha_{3}$

From this comparison, it is also inferred that the coefficient of eddy viscosity $\left(c_{\mu}\right)$ is a function of strain and rotation parameters. The strain parameter $(S)$ and rotation parameter $(\Omega)$ are defined in Eq. (12), as used in the previous studies of Pope ${ }^{9)}$, and Gatski and Speziale ${ }^{10)}$.

$S=\frac{k}{\varepsilon} \sqrt{\frac{1}{2} S_{i j} S_{i j}}, \quad \Omega=\frac{k}{\varepsilon} \sqrt{\frac{1}{2} \Omega_{i j} \Omega_{i j}}$

Many kinds of model functions have been proposed for the coefficient $c_{\mu}$. Most of them consider only strain parameter, and rotation parameter is neglected (such as, Cotton \& Ismail ${ }^{11)}$, Kato \& Launder ${ }^{12)}$ ). Craft et. al. ${ }^{13)}$ and Kimura \& Hosoda ${ }^{14)}$ consider one dominant parameter of two (either $S$ or $\Omega$ ). Recently, authors have proposed more generalized functional form for these coefficients considering the effect of both the parameters ${ }^{3), 4)}$. The proposed functional forms are as follows:

$c_{\mu}=\frac{c_{\mu o}\left(1+c_{n s} S^{2}+c_{n \Omega} \Omega^{2}\right)}{1+c_{d s} S^{2}+c_{d \Omega} \Omega^{2}+c_{d s \Omega} S \Omega+c_{d s 1} S^{4}+c_{d \Omega 1} \Omega^{4}+c_{d s \Omega 1} S^{2} \Omega^{2}}$

$c_{\beta}=c_{\beta o} \frac{1}{1+m_{d s} S^{2}+m_{d \Omega} \Omega^{2}}$

Table 1. Values of coefficients for $c_{\mu}$ and $c_{\beta}$

\begin{tabular}{lccccc}
\hline $\begin{array}{l}\text { Model } \\
\text { Const. }\end{array}$ & $c_{\mu 0}$ & $c_{n s}$ & $c_{n \Omega}$ & $c_{d s}$ & $c_{d s \Omega}$ \\
\hline Values & 0.09 & 0.0053 & 0.0065 & 0.008 & -0.003 \\
\hline & & & & & \\
\hline$c_{d \Omega}$ & $c_{d s l}$ & $c_{d \Omega l}$ & $c_{d s \Omega 1}$ & $m_{d s}$ & $m_{d \Omega}$ \\
\hline 0.004 & 0.00005 & 0.00005 & 0.00025 & 0.01 & 0.003 \\
\hline
\end{tabular}

Here, $c_{n s}, c_{n \Omega}, c_{d s}, c_{d \Omega}, c_{d s \Omega}, c_{d s l}, c_{d \Omega 1}, c_{d s \Omega 1}, m_{d s}$ and $m_{d \Omega}$ are the model constants. The functional form of $c_{\mu}$ used in the algebraic stress model by Gatski and Speziale ${ }^{10)}$ or Pope ${ }^{9)}$ can be obtained from the above equation simply neglecting some of the terms. Moreover, when the strain and rotation effects are neglected i.e. $S=\Omega=0, c_{\mu}$ becomes equal to the standard value of 0.09 . Neglecting the quadratic term, the model reduces to the standard $k-\varepsilon$ model.

If the effect of strain and rotation parameters on $c_{\beta}$ is neglected, Eq. 14 gives $\mathrm{c}_{\beta}=c_{\beta 0}$. The values of $c_{\beta 0}$ used in this study are given below:

$c_{1_{0}}=0.40, c_{2_{0}}=0, c_{3_{0}}=-0.13$

\subsection{Tuning of model constants and evaluation of model performance}

The values of model constants are given in Table 1 . The model constants in Eq. (13) are tuned considering the anisotropy of turbulence in plane shear layer, and that in Eq. (14) are tuned to satisfy the realizability conditions for 2D shear flows.

Further, the model is tested with some basic turbulent flows, such as turbulent jet with and without swirl (Ali et al. ${ }^{3)}$ ). The analytical results are compared to previous experiments, and the model constants are tuned to obtain the best fitted comparison. The swirl jet calculation explores the model's applicability for a flow field with high strain and rotation rate. The applicability of the model is examined for large scale vortices considering the spatial distribution and topological change of turbulent structures with singular 
points in an idealized vortex street (Ali et al. ${ }^{4)}$ ).

We also simulated the 3D flow field for compound open channel flows, and the performance of model is evaluated from the view point of mean flow and bed shear stress profiles, structure of secondary currents as well as considering the predictability of coherent vortices in the interface of main channel and flood plain (Ali et al. ${ }^{15}$ ).

\section{Simulation Details}

\subsection{Initial Conditions}

The q-vortex, for which the direct numerical simulation results are available $\left(\mathrm{Qin}^{6)}\right)$, is considered as initial condition of the vortex. Since the axial velocity of this vortex is not a function of axial distance but a function of radial distance, 2D numerical grid became applicable to simulate 3D flow field. The details of the flow field is given below.

Tangential velocity, $\frac{V_{\theta}}{V_{0}}=\frac{q_{0}}{r}\left(1-e^{-r^{2}}\right)$

Radial velocity, $\quad \frac{V_{r}}{V_{0}}=0$

Axial velocity, $\quad \frac{V_{z}}{V_{0}}=1-e^{-r^{2}}$

Here, $V_{0}$ is the scaling velocity, related to the initial mean tangential flow, defined as

$V_{0}=\frac{M_{p} \gamma}{q_{0}\left(1-e^{-r^{2}}\right)}$

$\mathrm{M}_{\mathrm{p}}$ is the initial DNS mach number $=0.0009$ and $\gamma=1.1209$ as used in Qin's simulation.

As discussed by Lessen et al. ${ }^{16)}$, the stability of q-vortex can be related to the value of swirl number q. They find that for any values of $q_{0}<\sqrt{2}$, the vortex is initially unstable and the addition or subtraction of a constant velocity to the axial velocity profile, or an inversion of a velocity deficit to a velocity excess, does not change the temporal stability of the vortex. The initial swirl number $\mathrm{q}_{0}$ is, thus, chosen to be unity to make the vortex initially unstable.

The time is non-dimensionalized by $\mathrm{T}=2 \pi r_{0} / V_{\theta m}$ at $\mathrm{t}=$ 0 . Here, $r_{0}$ is the radial distance where the tangential velocity contains the peak value $\left(V_{\theta m}\right)$ in the initial flow field (at $t=0$ ). The overall vortex behavior is found independent on the initial values of $k$ and $\varepsilon$. In this study, their values are given as $10^{-5}$ and $10^{-6}$ respectively.

\subsection{Flow domain and Computational schemes}

The governing equations for mean velocities and turbulent flows are discretized with the finite volume method based on a staggered grid system. For the momentum equation, convective and diffusive fluxes are approximated with QUICK and central difference schemes respectively. The hybrid central upwind scheme is used for $k$ and $\varepsilon$ equations. Time advancement is achieved by Adam-Bashforth scheme of second-order accuracy, in each equation. The basic equations are discretized as fully explicit forms and solved successively with the time increment in step by step. The pressure field is solved using iterative procedure at each time step.

Unsteady numerical simulations for 3-D velocity field are performed under the same hydraulic conditions of Qin's DNS computation. 2D numerical mesh with variable grid spacing is used with dense grid at centre and coarser towards the boundary. The computational domain consists of 100 grids in each of two lateral ( $x$ and $y$ ) directions. The size of domain is taken sufficiently large $(10 \mathrm{~m} \times 10 \mathrm{~m})$ to overcome the interference of boundary in the vortex decay process.

Cartesian grid is used for computation, and the results are finally presented in cylindrical coordinate using the geometric conversion.

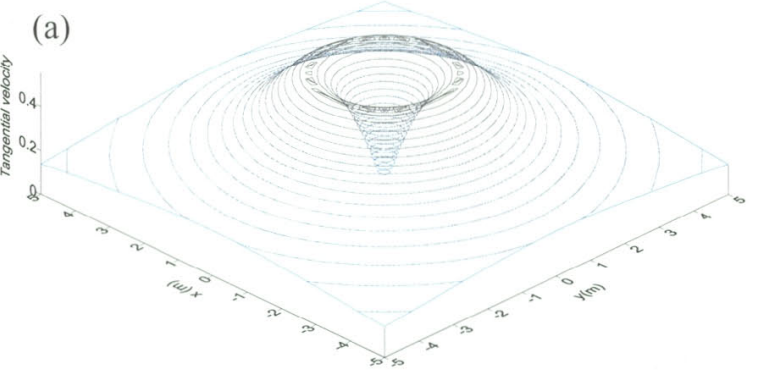

(b)
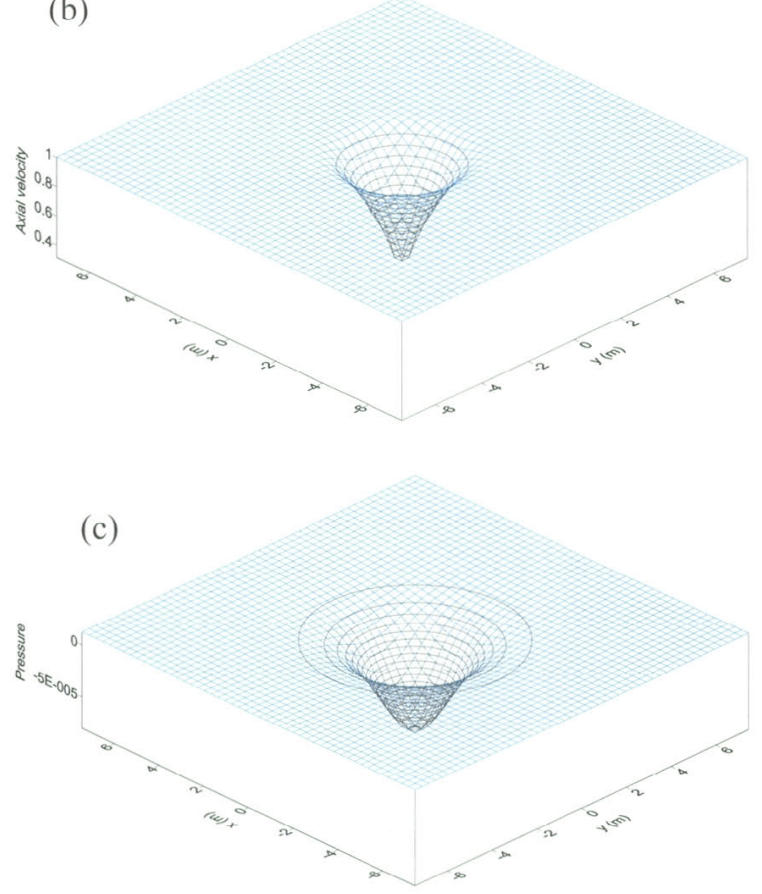

Fig. 1 Distribution of (a) Tangential velocity (b) Axial velocity and (c) pressure at $\mathrm{t}=3.72 \mathrm{~T}$ 


\section{Results}

The distribution of tangential and axial velocities as well as corresponding pressure distribution in the vortex field are shown in Fig.1, for simulation time $t=3.72 \mathrm{~T}$. The case of trailing vortex considered here contains the axial velocity with a minimum magnitude at vortex center (at center, $V_{z}=0$ at $\mathrm{t}=0$ ) that gradually increases in outward direction and became constant at a far distance from center. In the simulated results, the axial velocity in the center region is found to increase with time to reduce the gradient with far field. The tangential velocity also decays with time. The details of temporal change in flow characteristics are explained in next sections.

The pressure in a vortex is not uniformly constant with the radial distance. We know the distribution of pressure for a solid body rotation, for instance in a rotating box, is parabola $^{17)}$. The minimum pressure exists at center and increases towards the wall of the box. On the other hand, for a potential vortex the pressure increases from wall to outward distance in a concave manner. Therefore, the combination of these two gives the pressure distribution in a line vortex. The distribution of pressure in a trailing vortex is found similar to the line vortex regardless the presence of axial velocity. The figure shows that the pressure at vortex center is minimum and gradually increasing in outward direction and became constant at a far distance from center.

\subsection{Decay of vortex}

Figs. 2, 3 and 4 show the profiles of tangential velocity, circulation and axial velocity for different times calculated by using Non-linear $k-\varepsilon$ model. Figs. 5, 6 and 7 show those for standard $k-\varepsilon$ model. It is observed that the standard model shows faster decay of tangential and axial velocity than the non-linear model. Since the rotational effects of vortex were not captured by a standard $k-\varepsilon$ model, it predicted extremely rapid and strong turbulence growth and causes a rapid decay of tangential and axial velocity.

The circulation is zero at vortex center line and found to increase toward the radial direction that reached a free stream value at some radius outside the vortex core (at $r \gamma / r_{0}$ $>5$ ). A circulation overshoot is observed after the adjustment of initial conditions and when the vortex is well matured (at about $\mathrm{t}>3.0 \mathrm{~T}$ ). Qin's DNS simulation also shows a brief overshoot after the vortex adjusts itself to the unphysical initial conditions. Saffman ${ }^{18)}$ claimed that the circulation overshoot is a general feature of turbulent vortices. However Phillips ${ }^{5)}$ argued that the circulation overshoot is unlikely. Some research pointed out that the overshoot does not appear to be enough to produce a visible instability. Uberoi $^{19)}$ added that overshoot is possible if vortex diffusion rate is greater than that for a laminar vortex.

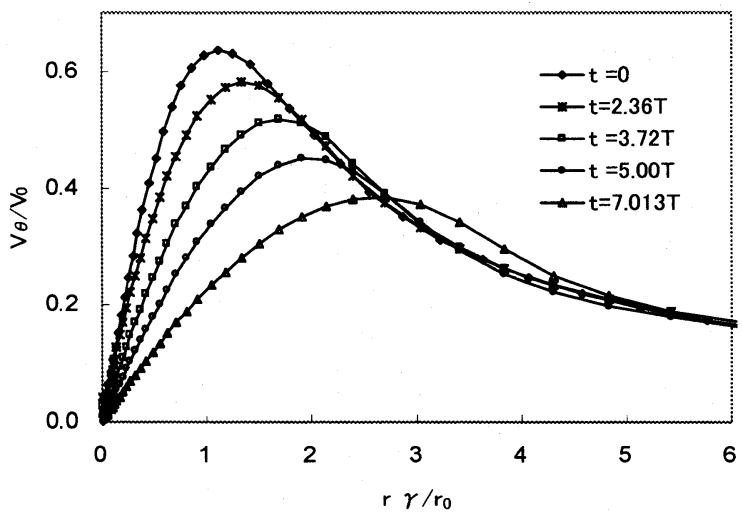

Fig. 2 Radial distribution of tangential velocity and its decay with time (Non-linear $k-\varepsilon$ model)

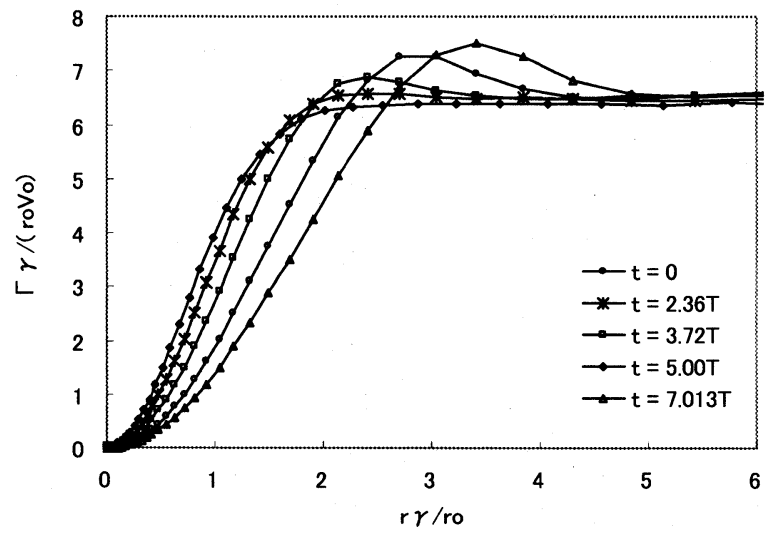

Fig. 3 Radial distribution of normalized circulation for different times (Non-linear $k-\varepsilon$ model)

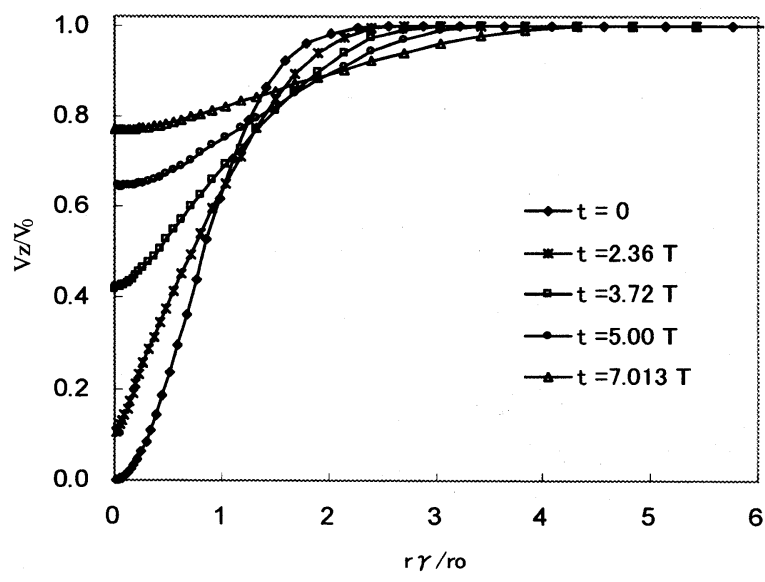

Fig. 4 Radial distribution of axial velocity and its decay with time (Non-linear $k-\varepsilon$ model)

In the tangential velocity, maximum decay occurs at the point of maximum velocity, while for axial velocity the maximum decay is at center of vortex. Fig. 8 compared the percentage change of axial velocity and peak tangential velocity with initial time. It is observed that the decay rate 
of axial velocity is much higher than tangential velocity. Since the vortex takes some time to adjust with initial condition, it is observed that the decay rate is small at initial times, approximately $\mathrm{t}<1.25 \mathrm{~T}$. For both the velocities, the high decay rate is observed in intermediate time period of $1.25 \mathrm{~T}>\mathrm{t}<4.25 \mathrm{~T}$. After that the decay slows down and the slope of trajectories shows to proceed towards minimum.

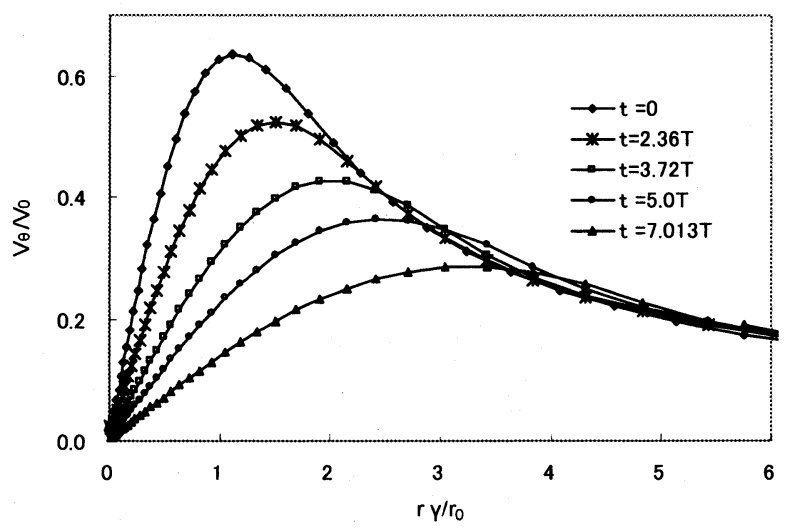

Fig. 5 Radial distribution of tangential velocity and its decay with time (Standard $k-\varepsilon$ model)

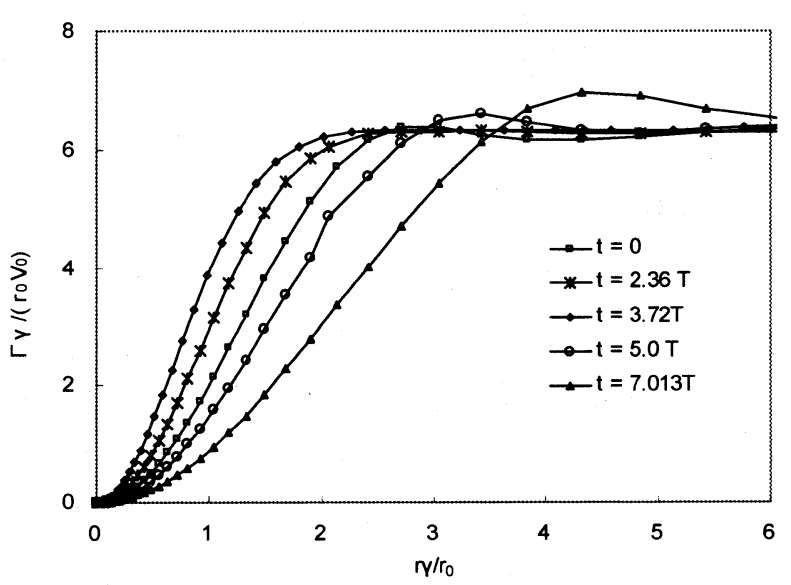

Fig. 6 Radial distribution of normalized circulation for different times (Standard $k-\varepsilon$ model)

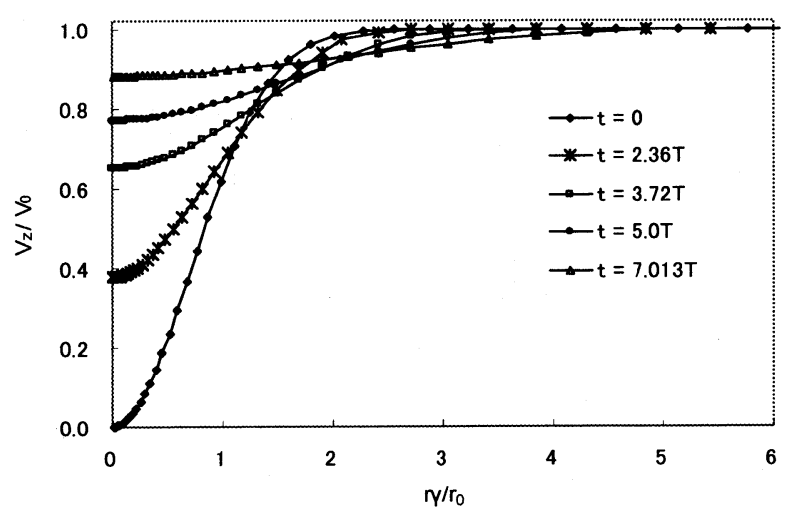

Fig. 7 Radial distribution of axial velocity and its decay with time (Standard $k-\varepsilon$ model)

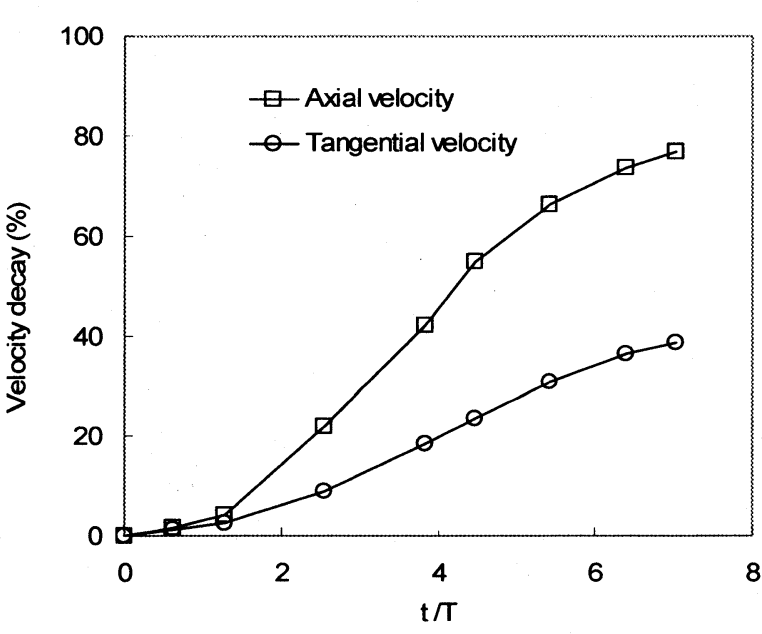

Fig. 8 Comparison between time decay of axial velocity and tangential velocity (Non-linear $k-\varepsilon$ model)

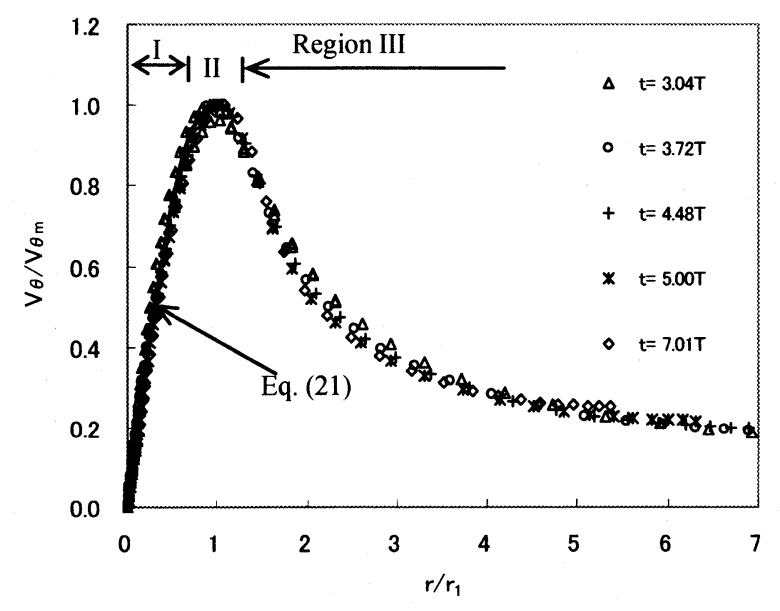

Fig. 9 Self-similar profile of circumferential velocity compared with Phillips' model.

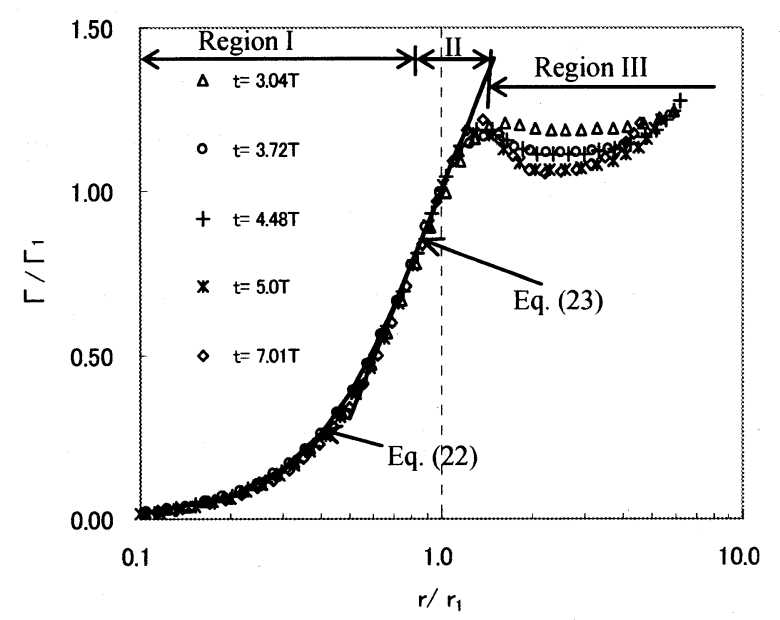

Fig. 10 Self-similarity in the circulation profile compared with Phillips' model 


\subsection{Self-similarity of profiles}

From dimensional analysis, Hoffmann \& Joubart ${ }^{7)}$ showed that for fully-developed flow

$\frac{V_{\theta}}{V_{\theta m}}=f\left(\frac{r}{r_{1}}\right) \quad$ or $\quad \frac{\Gamma}{\Gamma_{1}}=f\left(\frac{r}{r_{1}}\right)$

Here, $r_{l}$ is the radial distance of maximum tangential velocity $V_{\theta m} . \Gamma$ is the circulation defined as $\Gamma=2 \pi r V_{\theta}$, and $\Gamma_{1}$ is its value at a radial distance of $r_{l}$.

By plotting experimental results of $V_{\theta} / V_{\theta m}$ versus $r / r_{l}$, Phillips ${ }^{5)}$ showed that for a fully developed flow the data collapse in a single curve, and independent of the outer flow characteristics. Comparing with experiments, Phillips ${ }^{5)}$ proposed following equations to fit the self similar profile. Region-I:

$\frac{V_{\theta}}{V_{\theta m}}=1.7720\left(\frac{r}{r_{1}}\right)-1.0467\left(\frac{r}{r_{1}}\right)^{3}+0.2747\left(\frac{r}{r_{1}}\right)^{5}$

$\frac{\Gamma}{\Gamma_{1}}=1.7720\left(\frac{r}{r_{1}}\right)^{2}-1.0467\left(\frac{r}{r_{1}}\right)^{4}+0.2747\left(\frac{r}{r_{1}}\right)^{6}$

Logarithomic part of Region-II:

$\frac{\Gamma}{\Gamma_{1}}=\ln \left(\frac{r}{r_{1}}\right)+1$

In Fig. 9, it is observed that for tangential velocity the self similarity solution embraces all the regions I, II and III. Here the profiles are shown for $\mathrm{t}>3.0 \mathrm{~T}$. Eq (21) proposed by Phillips ${ }^{5)}$ is perfectly fitted with the simulation results by non-linear $k-\varepsilon$ model. For $\mathrm{t}<3$.0T, region I and II are found to show self similarity, but region III does not collapse with the data shown in Fig. 9. Therefore, region III shows the similarity form only for a particular flow conditions and in the fully developed flow case.

Fig. 10 shows the self-similar circulation profile. It is observed that although the simulated results for region I and region II collapse in a single curve, region III does not show the self similarity form. Eqs. (22) and (23) also show excellent fitting with the simulated data. From previous studies it is found that region I is weakly dependent on initial and hydraulic conditions. Phillips noted that this self-similar profile exists in the region I and II, regardless the presence of axial velocity component. Present result also support the argument and found that this region shows self-similarity form for all the times including $\mathrm{t}<3.0 \mathrm{~T}$. The logarithmic region near $r / r_{l}=1$ is reported as truly universal region. Dissimilarity in region III observed in Figure is also reported in previous studies. Due to circulation overshoot, there does not seem to be any scope for the self-similarity solution in region III.

\subsection{Instability vortices}

The non-linear model shows the occurrence of instability in a well grown vortex field (i.e. at $\mathrm{t}>3.0 \mathrm{~T}$ for this case) after the self-similar form of flow field is attained. Fig. 11 shows the flow velocity vectors and the instability vortices in the flow field at $\mathrm{t}=3.1 \mathrm{~T}$ and $7.01 \mathrm{~T}$. Note that the instability vortex field is calculated by subtracting the mean velocity field from the main flow field. Phillips' multilayered structure is shown in the figure. Due to tangential velocity gradient between region I and II, as well as between II and III, instability vortices are found to form in those regions. Although at lower time $(\mathrm{t}=3.1 \mathrm{~T})$ the vortices between region I and II are not visible, they are clearly observed at $\mathrm{t}=7.01 \mathrm{~T}$. In these figures, the instability vortices are observed at a radial distance of about $0.7 r_{l}$ and $1.2 r_{l}$ respectively (locations are shown as dotted circular lines).

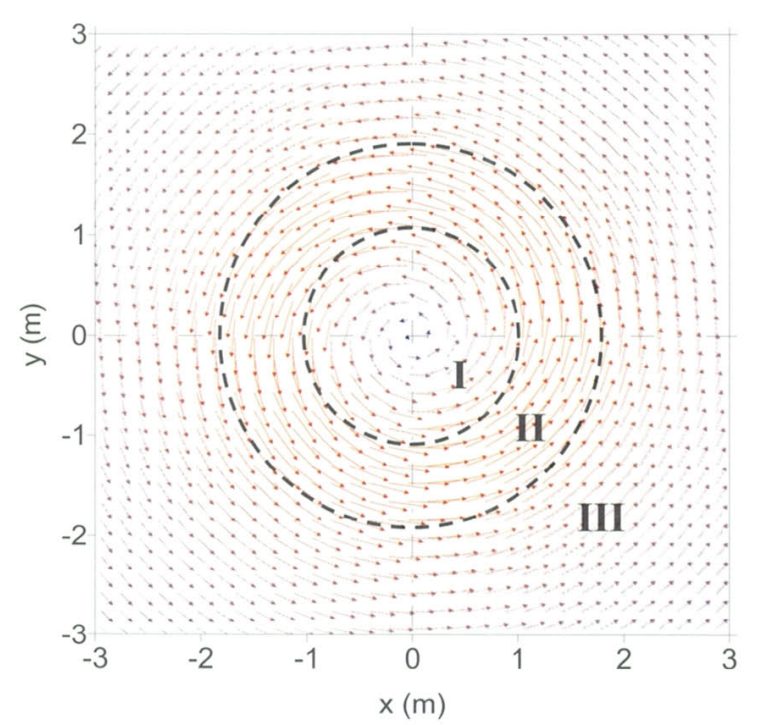

(a) Calculated velocity vector of the flow field at $t=3.1 \mathrm{~T}$

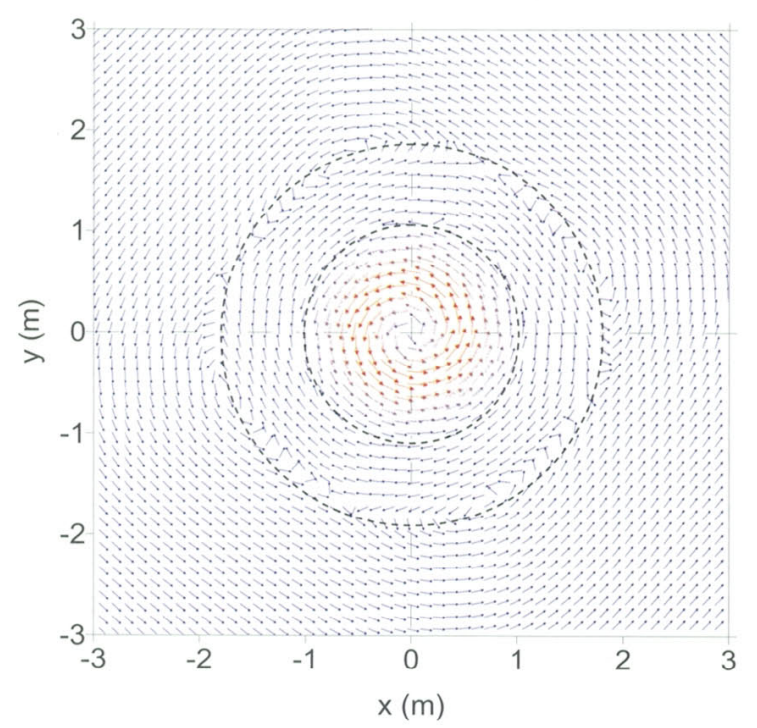

(b) Instability vortices in the flow field at $\mathrm{t}=3.1 \mathrm{~T}$ 


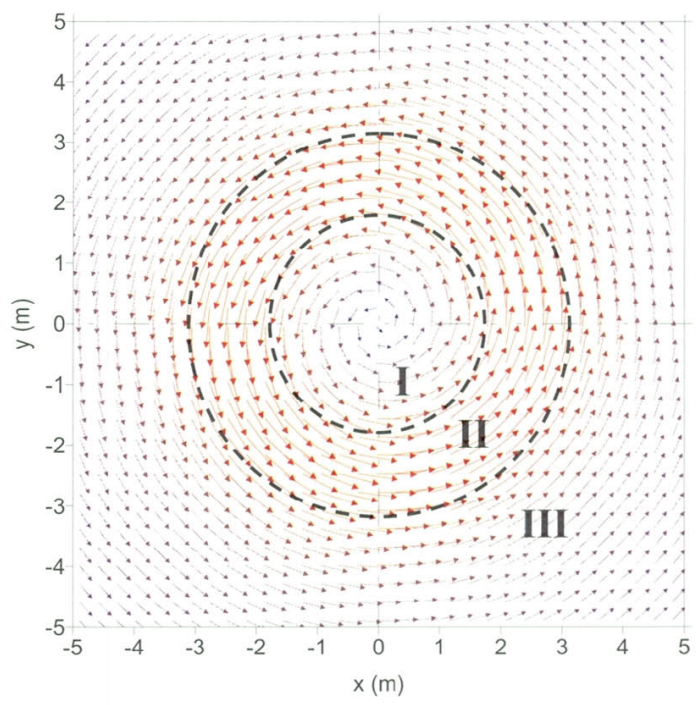

(c) Calculated velocity vector of the flow field at $t=7.01 \mathrm{~T}$

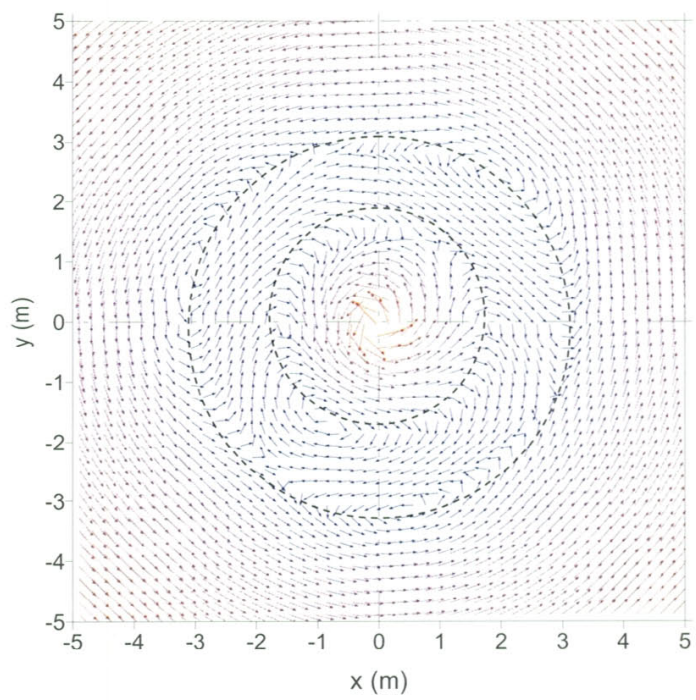

(d) Instability vortices in the flow field at $t=7.01 \mathrm{~T}$

Fig. 11 Non-linear $k-\varepsilon$ model prediction of velocity vectors and instability vortices in the flow field

\subsection{Turbulence growth with time}

Production of turbulence is due to the tangential and axial velocity gradients. In the vortex lifetime, five different growth/decay rates of turbulent kinetic energy as reported in DNS calculation ${ }^{6)}$ is also observed in the simulation of non-linear $k-\varepsilon$ model. Fig. 12 shows the growth/decay of turbulence kinetic energy with approximated five different time zones. Initially (zone (i)), the vortex changes very slowly, as it requires to adjust with any unphysical nature of initial conditions. In time zone (ii), an exponential growth of turbulent kinetic energy is observed. The growth slows down in the next time period of zone (iii). It is observed that the decay of axial velocity gradient is significantly high in time zone (ii) and (iii). The turbulent kinetic energy reaches in its peak value and remains about constant throughout a short period of zone (iv). It reveals that in this stabilization period the flow field became saturated and cannot support additional turbulence. Finally, the decay of turbulence is started as predicted by most of the previous researches (such as, Uberoi ${ }^{19)}$, Bachelor ${ }^{20)}$, etc.). The logarithmic plot shows that the decay rate in zone (v) is much slower than the growth rate in zone (ii).

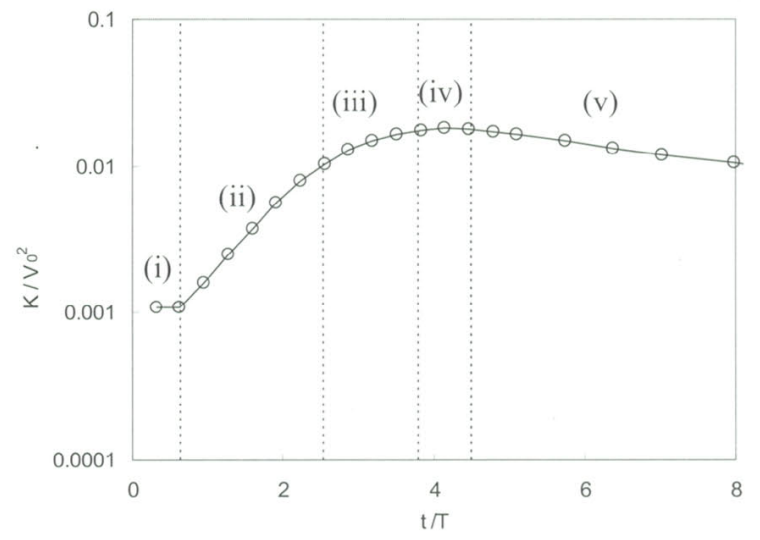

Fig. 12 Growth of turbulent kinetic energy at $r \gamma / r_{0}=0.7$ with different time periods (Non-linear $k-\varepsilon$ model)

Fig. 13 shows the radial distribution of turbulent normal stresses $\left(\mathrm{R}_{\mathrm{ii}}\right)$ in axial, radial and tangential directions for different times; those are noted as $\overline{u_{z} u_{z}}, \overline{u_{r} u_{r}}$ and $\overline{u_{\theta} u_{\theta}}$ respectively. Here, the radial distance is normalized by $r_{l}$ $=r_{0}(t)$, which is the radial distance of maximum tangential velocity. For a round jet in a stagnant surroundings, $\overline{u_{z} u_{z}}>\overline{u_{\theta} u_{\theta}} \geq \overline{u_{r} u_{r}}$ over the radius of jet (Ali et al. ${ }^{3)}$, Wygnanski \& Fielder ${ }^{21)}$ ). However, in the matured stage of a trailing vortex, the turbulent normal stresses show two types of anisotropy depending on the radial distance. Inside the vortex core $\left(r / r_{1}<1\right)$ up to certain distance from center, the relation is $\overline{u_{r} u_{r}} \geq \overline{u_{\theta} u_{\theta}}>\overline{u_{z} u_{z}}$; and beyond that distance the stresses follows $\overline{u_{r} u_{r}}>\overline{u_{z} u_{z}}>\overline{u_{\theta} u_{\theta}}$. The latter case is the situation where turbulence production is significantly less than diffusion, such as in the central region of an axisymmetric wake. These anisotropic behaviors of turbulence are also reported in previous studies by Devenport et al. ${ }^{22)}$ and Phillips \& Graham ${ }^{23)}$.

In the growth stage of time zone (ii), all the normal stresses are found to increase simultaneously. In Fig. 13, the distribution of turbulent normal stresses are shown for different times $(\mathrm{t}=3.72 \mathrm{~T}, 5.0 \mathrm{~T}$ and $7.013 \mathrm{~T})$, which are belongs to time zones (iii), (iv) and (v), respectively. Comparison of turbulent intensities between time zones (iii) and (iv), it is observed that only the axial velocity 
component shows significant decay. On the other hand, comparison between (iv) and (v), shows the simultaneous decay of turbulent intensities in all the velocity components.

It is observed that at the center of vortex (i.e. at $r=0$ ), $\overline{u_{\theta} u_{\theta}}=\overline{u_{r} u_{r}}$ throughout the simulation time. It confirms the axisymmetric condition of the flow field.
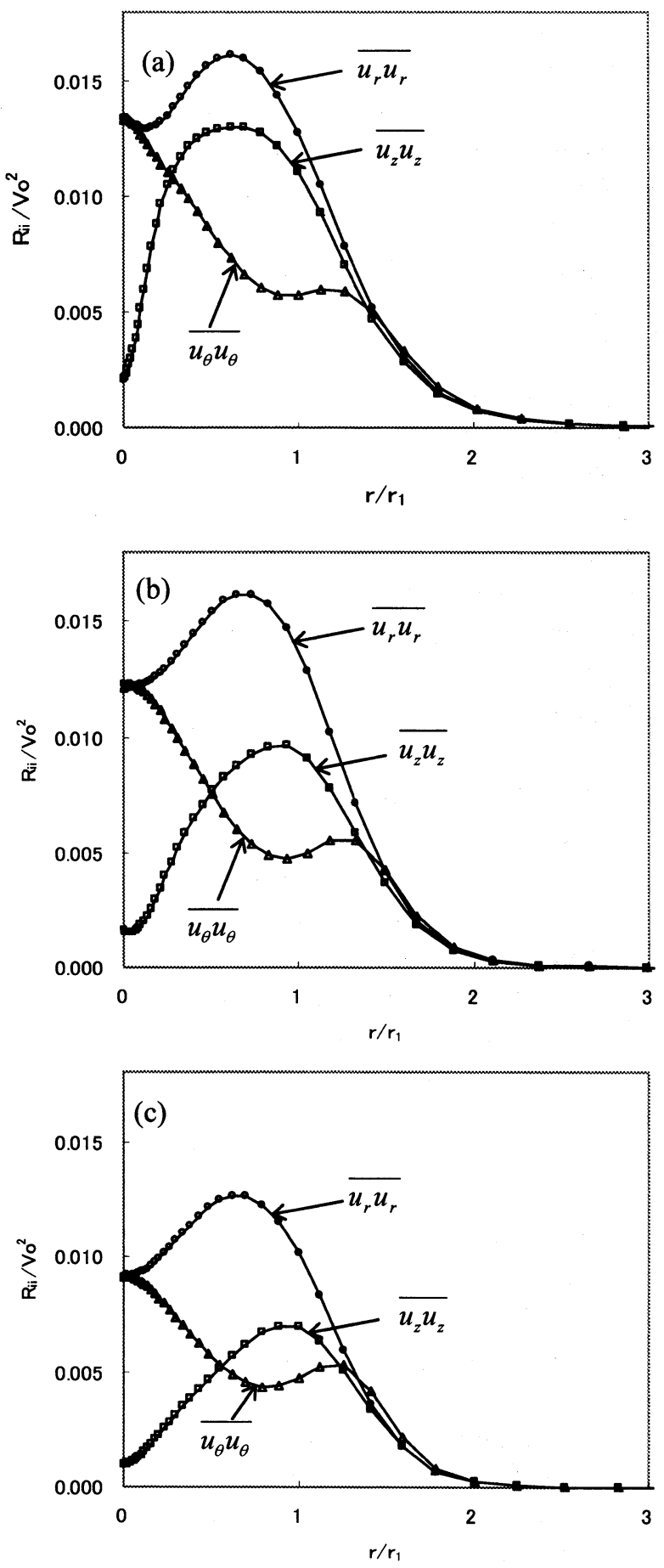

Fig. 13 Radial distribution of turbulent intensities in three directions at (a) $t=3.72 \mathrm{~T}$ (b) $\mathrm{t}-5.0 \mathrm{~T}$ (c) $\mathrm{t}-7.013 \mathrm{~T}$

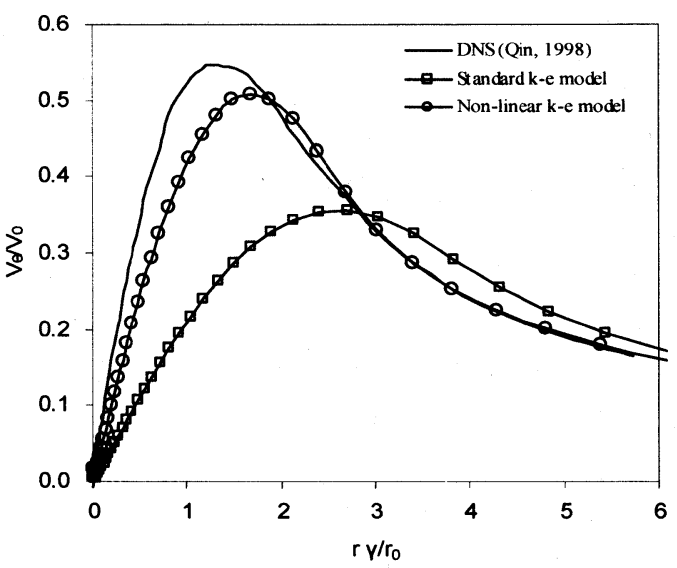

Fig. $14 k-\varepsilon$ model prediction of tangential velocity profile compared with Qin's ${ }^{6}$ DNS results $(t=3.72 T)$

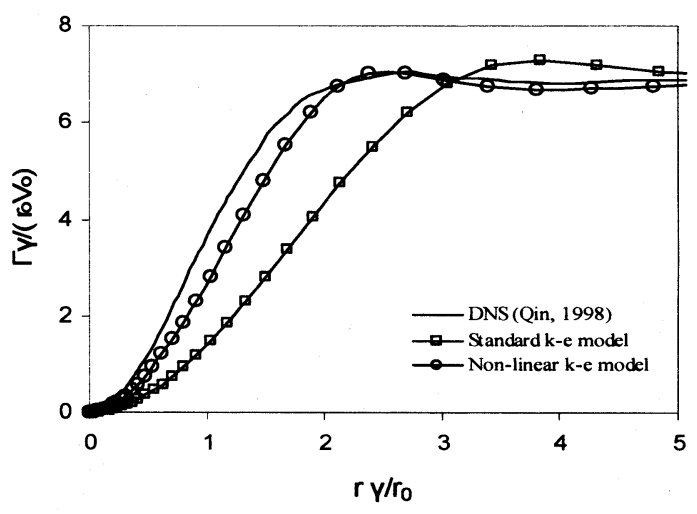

Fig. $15 k-\varepsilon$ model prediction of circulation profile compared with Qin's $\left.{ }^{6}\right)$ DNS results $(t=3.72 T)$

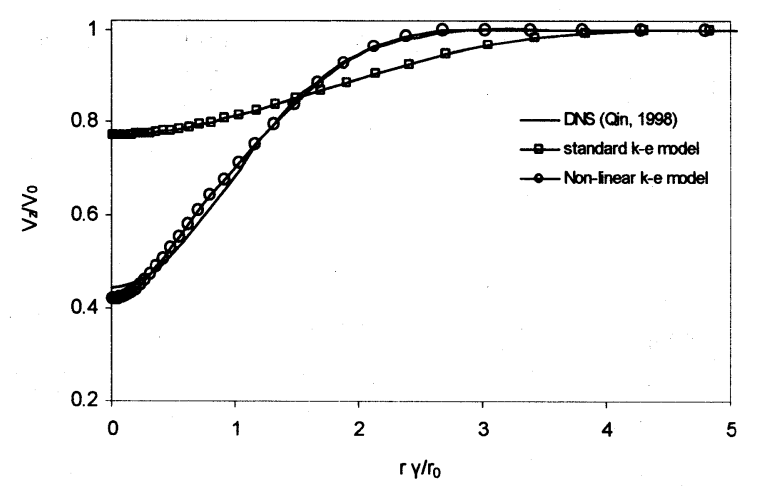

Fig. $16 k-\varepsilon$ model prediction of axial velocity profile compared with Qin's ${ }^{6)}$ DNS results $(t=3.72 T)$

\subsection{Comparison with Qin's DNS data}

The circumferential velocity, the circulation and the axial velocity are compared with the DNS calculation of $\mathrm{Qin}^{6)}$ at time $\mathrm{t}=3.72 \mathrm{~T}$ as shown in Figs. 14,15 and 16 respectively. At this stage the vortex already overcome the effect of initial conditions and shows self-similar behavior. Also, the turbulent flow field became saturated and 
stabilized gaining a peak value of turbulent kinetic energy after passing its growth periods. From Figs. 14 to 16 , it is observed that the non-linear model shows well comparison with DNS data. Although the axial velocity decay is fitted well, the magnitude and position of maximum tangential velocity shows slightly faster decay than DNS data. A very high decay rate is observed for the standard model in comparison to the non-linear model and DNS simulation. Such discrepancy also observed in the comparison of circulation profile. Comparing the turbulence kinetic energy in Fig. 17, it is observed that the non-linear model shows much better comparison over standard model. However, the model slightly over-predicts the kinetic energy near its maximum point, and it is the cause of decay of tangential velocity slightly faster than DNS data as observed in Fig.14. Further refinement of the model, such as fine tune of model constants and/or addition of 3rd order terms, are necessary to minimize this small disagreement.

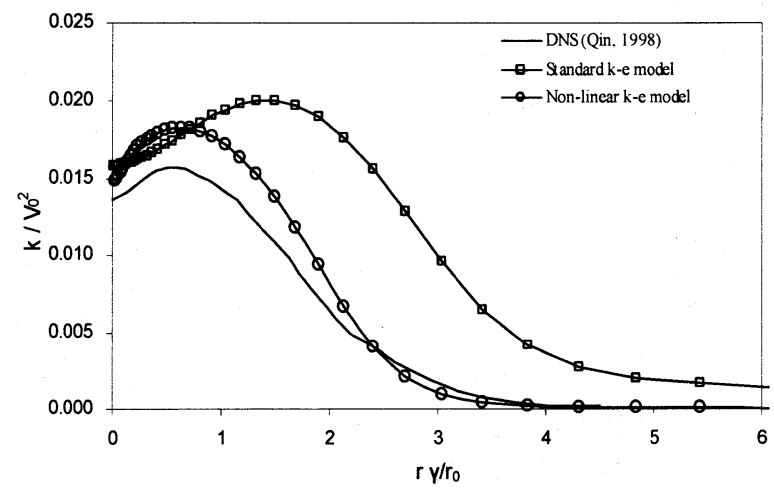

Fig. $17 k-\varepsilon$ model prediction of turbulent kinetic energy with Qin's ${ }^{6)}$ DNS results $(t=3.72 T)$

\section{Conclusion}

The unsteady numerical simulations are performed for an isolated turbulent axial vortex using standard and non-linear $k-\varepsilon$ models. Since the rotational effects of vortex were not captured by a standard $k-\varepsilon$ model, it predicted extremely rapid and strong turbulence growth and causes a rapid decay of tangential and axial velocities. However, the non-linear model shows good agreement with DNS data. For the tangential velocity, the self similarity solution embraces regions I, II (defined by Phillips) for all the time periods, however region- III collapse in a single curve only for fully developed flow. Phillips' model equations for similarity solution of velocity and circulation profiles are perfectly fitted with the simulated results. Circulation overshoot is observed in the well grown vortex field. Due to this overshoot, the self similarity solution seems to be not possible in region III.

It is observed that the decay rate of axial velocity is much higher than tangential velocity. Since the vortex takes some time for initial adjustments, it is observed that the decay rate is small at initial times, approximately $\mathrm{t}<1.25 \mathrm{~T}$. For both the velocities, the high decay rate observed in intermediate time period of $1.25 \mathrm{~T}>\mathrm{t}<4.25 \mathrm{~T}$. After that the decay slows down, and the slope of decay shows to proceed towards minimum.

Five different time periods are observed in the growth/decay profile of turbulence kinetic energy. Initially the vortex changes very slowly to adjust with initial conditions, then an exponential growth is observed that slows down before gaining its peak value. The peak value of turbulent kinetic energy remains about constant for a short period that finally follows the period of turbulence decay.

After the maturity of vortex, the turbulent normal stresses show two types of anisotropic behavior depending on the radial distance. Although the radial component is always greater than other two, the tangential component is greater near the center and smaller around the region of maximum tangential velocity in comparison to axial component. The decay rate of energy after the period of stabilization is much slower than the growth rate in the time zones before the stabilization. The decay of velocity field slows down as the turbulence decay period starts.

\section{References}

1) Jaw, S.Y. and Chen, C. J., Present status of second order closure turbulence models, I: overview. $J$. Engg. Mech. ASCE., Vol. 124, pp. 485, 1998.

2) Rodi, W., Turbulence models for environmental problems, in Prediction Methods for Turbulent Flows (edited by W. Kollmann), pp.259, 1979.

3) Ali, M. S., Hosoda, T., Kimura, I. and Onda, S., Approximate solution of an axisymmetric Swirling jet using non-linear $k-\varepsilon$ model with consideration of realizability, Journal of Applied Mechanics, JSCE, Vol. 9, pp. 821-832, 2006.

4) Ali, M. S., Hosoda, T. and Kimura, I., A non-linear $k-\varepsilon$ model to predict the spatial change of turbulent structures in large scale vortices, Journal of Applied Mechanics, JSCE, Vol. 10, pp. 723-732, 2007.

5) Phillips, W.R.C, The turbulent trailing vortex during roll-up, J. Fluid. Mech. 105, pp. 451-467, 1981.

6) Qin, J. H., Numerical simulation of a turbulent axial vortex, Phd thesis, Purdue University, USA, 1998.

7) Hoffmann, E.R. and Joubart, P.N., Turbulent line Vortices, J. Fluid. Mech. 16, pp. 423-439, 1963.

8) Yoshizawa, A., Statistical analysis of the deviation of the Reynolds stress from its eddy viscosity representation, Phys. Fluids, 27, pp.1377-1387, 1984.

9) Pope, S. B., A more general effective viscosity 
hypothesis, J. Fluid Mech. 72, pp.331-340, 1975.

10) Gatski, T. B. and Speziale, C. G., On explicit algebraic stress models for complex turbulent flows, J. Fluid Mech. 254, pp.59-78, 1993.

11) Cotton, M. A. and Ismail, J.O., Development of a two equation turbulence model with reference to a strain parameter, Proc. $5^{\text {th }}$ IAHR Conf. on Refined-flow Modeling and Measurement, Paris, pp. 117-124, 1993.

12) Kato, M. and Launder, B.E., The modeling of turbulent flow around stationary and vibrating square cylinder, Proc. $9^{\text {th }}$ Symp. on Turbulent ShearFlows, Kyoto Vol. 1, 10-4-4 10-46, 1993.

13) Craft, T.J., Launder, B.E. and Suga, K., Extending the applicability of eddy viscosity models through the use of deformation invariants and non-linear elements. Proc. $5^{\text {th }}$ IAHR Conf. on Refined-flow Modeling and Measurement, Paris. 125, 1993.

14) Kimura, I. and Hosoda, T., A non-linear $k-\varepsilon$ model with realizability for prediction of flows around bluff bodies, Int. J. Numer. Meth. Fluids. 42, pp.813-837, 2003.

15) Ali, M. S., Hosoda, T. and Kimura, I., Unsteady RANS computations of Compound channel flows with large scale vortices and secondary currents, $5^{\text {th }}$ International Symposium on Environmental Hydraulics, ASU, Tempe, Arizona, 2007.
16) Lessen, M., Singh, P.J. and Paillet, F., The stability of a trailing line vortex. Part I: Inviscid theory, J. Fluid. Mech. 63, pp. 753-763, 1974.

17) Lugt, H. J., Vortex flow in nature and technology, John-wiley \& Sons, USA (ISBN 0-471-86925-2), 1983.

18) Saffman, P.G., The structure and decay of turbulent trailing vortices, Arch. Mechanics, 26(3), pp. 423-439, 1974.

19) Uberoi, M.S., Mechanisms of decay of laminar and turbulent vortices. J. Fluid. Mech. 90(2), pp. 241-255, 1979.

20) Bachelor, G. K., Axial flow in trailing line vortices, $J$. Fluid Mech. 20(4), pp.645-658, 1964.

21) Wygnanski, I. and Fielder, H., Some measurements in the self-preserving jet, J. Fluid Mech. 38, pp.577-612, 1969.

22) Devenport, W. J., Rife, M.C., Liapis, S.I., and Follin, G.J., The structure and development of a wing-tip vortex, J. Fluid. Mech. 312, pp. 67-106, 1996.

23) Phillips, W.R.C. and Graham, J.A.H, Reynolds stress measurement in a turbulent trailing vortex, J. Fluid. Mech. 147, pp. 353-371, 1984.

(Received: April 14, 2008) 